\title{
Pseudotumor from a Metal-on-Metal Hip
}

TAKUJI IWAMOTO, MD, PhD; KATSUNORI IKARI, MD, PhD; SHIGEKI MOMOHARA, MD, PhD, Institute of Rheumatology, Tokyo Women's Medical University, 10-22 Kawada, Shinjuku, Tokyo 162-0054, Japan. Address correspondence to Dr. Ikari; E-mail: kikari@ior.twmu.ac.jp. J Rheumatol 2011;38:2265; doi:10.3899/jrheum.110806

Pseudotumors in the pelvis, thigh, and gluteal regions after metal-on-metal hip implants can occur as complications of the surgery ${ }^{1,2}$. The tumors can be caused by a metal hypersensitivity reaction associated with metal wear debris.

A 64-year-old woman with systemic lupus erythematosus who underwent total hip arthroplasty (THA) 3 years before presented with a tender mass on her left thigh, about $30 \mathrm{~mm}$ in diameter. The THA had been done using an Encore Medical (now DJO Surgical, Austin, TX, USA) Metal/Metal $\mathrm{CoCr}$ acetabular liner, a metal liner enclosed with a polyethylene dome. Although the patient felt mild left thigh pain, her walking ability was almost unaffected and radiographs revealed no obvious prosthesis failure on her left hip. Magnetic resonance imaging (T2-weighted) showed a mixed-intensity mass (proximal half was T2 low-intensity and distal half was T2 high-intensity) penetrating the fascia lata (Figure 1). Surgical resection was performed and intraoperative findings revealed the synovial tissue stained with metallosis (Figure 2). The synovial fluid and tissue cultures were negative for infection. Histologic examination of the synovial tissue showed inflammatory granulation tissue with metallosis and phagocytosis of polyethylene particles (Figure 3 ). The extraarticular tumor indicated the metallosis of the hip without any remarkable radiographic and physical symptoms. Because the tumor recurred 3 months after the tumor resection, revision THA (isolated acetabular liner exchange) was performed using a polyethylene liner, and no tumor recurrence was observed for 2 years after revision surgery.

Pseudotumors after metal-on-metal hip implants have

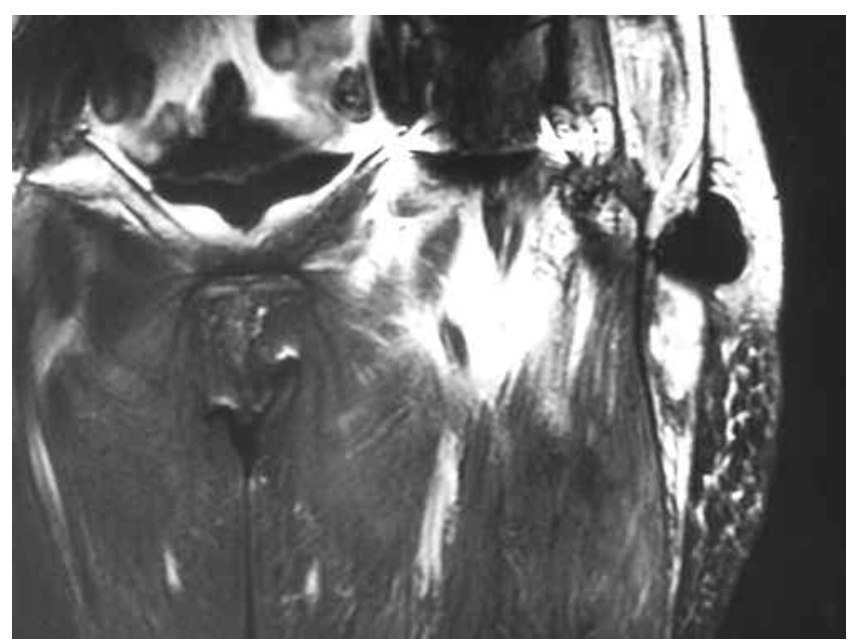

Figure 1. MRI scan shows a mixed-intensity mass penetrating the fascia lata.

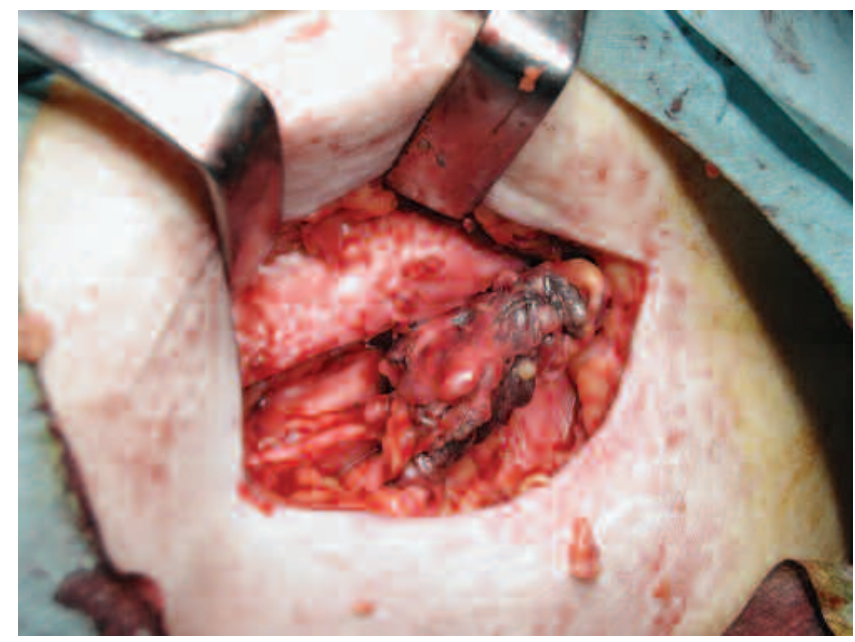

Figure 2. Surgical resection revealed synovial tissue stained with metallosis.

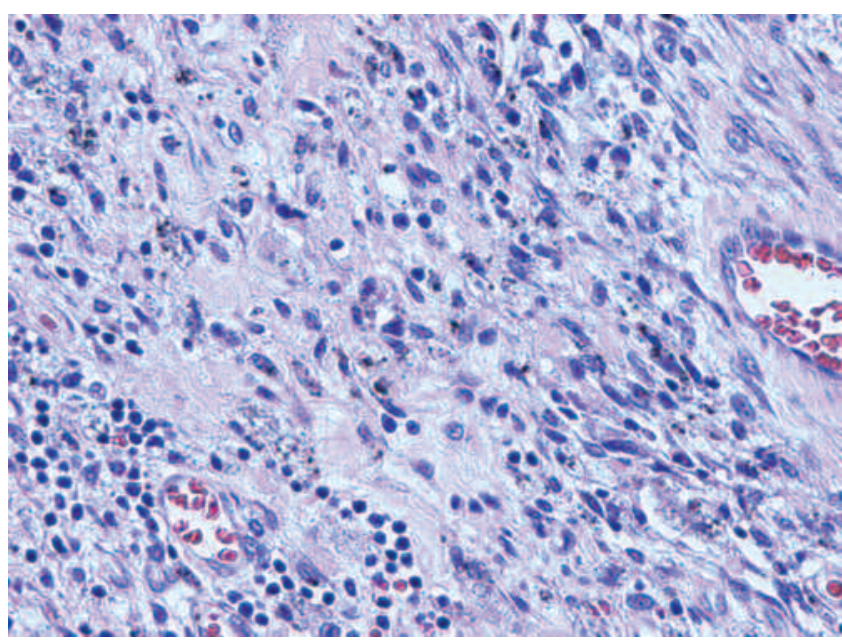

Figure 3. Histologic examination of the synovial tissue showed inflammatory granulation tissue with metallosis and phagocytosis of polyethylene particles.

been increasingly reported ${ }^{1,2}$. These complications of the surgery can result from a metal hypersensitivity reaction associated with metal wear debris from the implant.

\section{REFERENCES}

1. Canadian Hip Resurfacing Study Group. A survey on the prevalence of pseudotumors with metal-on-metal hip resurfacing in Canadian academic centers. J Bone Joint Surg Am 2011;93 Suppl 2:118-21.

2. Leigh W, O'Grady P, Lawson EM, Hung NA, Theis JC, Matheson J. Pelvic pseudotumor: an unusual presentation of an extra-articular granuloma in a well-fixed total hip arthroplasty. J Arthroplasty 2008;23:934-8. 